\title{
Anisotropic Isometric Fluctuation Relations in experiment and theory on a self-propelled rod
}

\author{
Nitin Kumar ${ }^{1}$, Harsh Soni ${ }^{1,2}$, Sriram Ramaswamy ${ }^{2, *}$ and A.K. Sood ${ }^{1}$ \\ ${ }^{1}$ Department of Physics, Indian Institute of Science, Bangalore 560 012, India and \\ ${ }^{2}$ TIFR Centre for Interdisciplinary Sciences, Tata Institute of Fundamental Research, \\ 21 Brundavan Colony, Osman Sagar Road, Narsingi, Hyderabad 500 075, Indid*
}

(Dated: October 4, 2018)

\begin{abstract}
The Isometric Fluctuation Relation (IFR) [P.I. Hurtado et al., PNAS 108, 7704 (2011)] relates the relative probability of current fluctuations of fixed magnitude in different spatial directions. We test its validity in an experiment on a tapered rod, rendered motile by vertical vibration and immersed in a sea of spherical beads. We analyse the statistics of the velocity vector of the rod and show that they depart significantly from the IFR of Hurtado et al. Aided by a Langevin-equation model we show that our measurements are largely described by an anisotropic generalization of the IFR [R. Villavicencio et al., EPL 105, 30009 (2014)], with no fitting parameters, but with a discrepancy in the prefactor whose origin may lie in the detailed statistics of the microscopic noise. The experimentally determined Large-Deviation Function of the velocity vector has a kink on a curve in the plane.
\end{abstract}

PACS numbers: 45.70. -n, 05.40.-a, 05.70.Ln, 45.70.Vn

Fluctuation relations (FR) go beyond the second law of thermodynamics by quantifying the relative probability of short-time, small-scale entropy-consuming events 1]. Experimental evidence for their validity is surprisingly widespread [2 5], even in non-thermal noisy systems nominally outside their realm of applicability [6 11$]$. A more stringent symmetry property of thermal systems, the Isometric Fluctuation Relation (IFR) [12], has been derived recently for isotropic systems, describing the relative probabilities of observing currents of equal magnitude in different directions, not necessarily diametrically opposed as in the standard FR [1].

In this Rapid Communication we present the first experimental observations of behaviour consistent with the IFR, on a macroscopic, fore-aft asymmetric rod which executes self-propelled [13 18] motion through a background of non-motile spheres. Our main results are as follows: (i) Our anisotropic experimental system deviates substantially from the predictions of [12]. (ii) We show that the symmetry properties of the LargeDeviation (LD) function correspond to the Anisotropic IFR of [19], with which we are able to show a parameterfree agreement through a simplified description based on an ansotropic single-particle Langevin equation. There remains a $20 \%$ discrepancy in the prefactor whose origin, we speculate, could lie in non-Gaussianity in the microscopic noise, not accounted for in our Langevin model. (iii) We find that the measured LD Function of the velocity vector exhibits a kink where the velocity component along the rod axis vanishes. We speculate why these behaviours should arise in an apparently nontime-reversible system.

In our experimental setup [16] a single geometrically polar brass rod, $4.5 \mathrm{~mm}$ long with diameter $1.1 \mathrm{~mm}$ at the thick end is placed amidst a monolayer of spherical aluminium beads of diameter $0.8 \mathrm{~mm}$ (Fig. 1(a)). The beads lie on an circular aluminium plate $13 \mathrm{~cm}$ in diameter, covered by a glass lid at $1.2 \mathrm{~mm}$ above the surface, thus forming a confined two-dimensional system. The bead area fraction, based on their projected images, is 0.83. A permanent-magnet shaker (LDS V406-PA 100E) drives the plate sinusoidally in the vertical direction with amplitude $a_{0}$ and frequency $f=200 \mathrm{~Hz}$, corresponding to dimensionless shaking strength $\Gamma \equiv a_{0}(2 \pi f)^{2} / g=6.5$, where $g$ is the acceleration due to gravity. The rod transduces the vibration into predominantly forward motion as indicated by the arrow in Fig. 1(a) 13, 14, 16]. The bead medium is both athermal noise source and obstacle course for the motion of the polar rod (see Supplementary Video). A high-speed camera (Redlake MotionPro $\mathrm{X} 3$ ) records the dynamics of the particle at a rate of 50 frames per second. A typical experimental snapshot is shown in Fig. 1(a). The images were analysed in ImageJ [20] to calculate the instantaneous in-plane position $\mathbf{R}(t)$ and orientation, i.e., the unit vector $\hat{\mathbf{n}_{\|}}(t)$ from the thick to the thin end of the rod of the polar rod, and its two-dimensional velocity vector $\mathbf{v}(t)$ at time $t$ defined as the discrete time derivative of $\mathbf{R}(t)$ between successive frames. Note that $\mathbf{v}(t)$ is already coarse-grained in time with respect to the true microscopic velocity. The plate vibrates at 4 times the frame rate, and the collisions of the rod with plate, lid and beads take place at irregular instants.

We resolve $\mathbf{v}(t)$ into components $\left(v_{\|}(t), v_{\perp}(t)\right)=(\mathbf{v}(t)$. $\left.\hat{\mathbf{n}_{\|}}(t), \mathbf{v}(t) \cdot \hat{\mathbf{n}_{\perp}}(t)\right)$ along and transverse to $\hat{\mathbf{n}_{\|}}(t)$ [Fig. [1(e)]. In earlier work [16] on this system we studied the statistics of $v_{\|}$alone and found a large-deviation function with a kink at zero as predicted in several theoretical treatments of forced Brownian motion amidst periodic obstacles 21 24]. The results of [16] encourage 


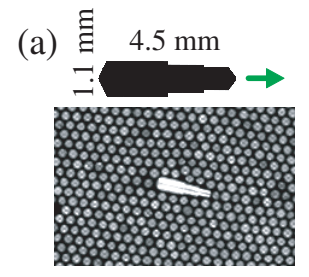

(b)
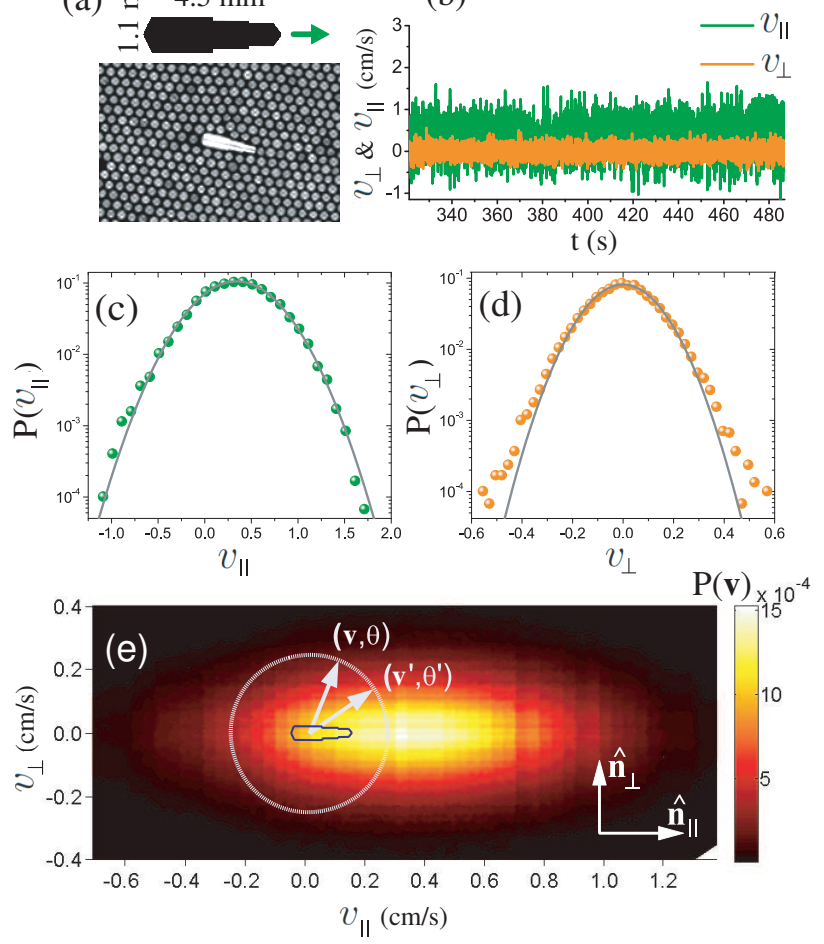

FIG. 1: (a) Geometry of the polar particle: the thick arrow along the particle's axis indicates the mean direction of "self-propelled" motion and typical experimental screen-shot depicting the particle moving its way through the crystalline medium consisting of 0.8 $\mathrm{mm}$ aluminium beads in a confined two-dimensional cell. (b) Time-series of velocity components $v_{\|} \& v_{\perp}$ of the particle. Positive direction is along the green arrow in (a). Probability distributions of $v_{\|}$, (c) and $v_{\perp}$, (d) with solid lines showing a Gaussian fit. (e) Resulting probability distribution of velocity $\mathbf{v} \equiv v_{\|} \hat{\mathbf{n}_{\|}}+v_{\perp} \hat{\mathbf{n}_{\perp}}$ of the particle. Circle shows a constant-velocity contour and the arrows representing isometric vectors subtending angles $\theta \& \theta^{\prime}$ with the horizontal axis.

us to further probe its nonequilibrium fluctuations and look for a possible IFR. Fig. 1(b) shows the instantaneous time-series of $v_{\|}$, whose nonzero mean signals systematic "self-propulsion", and $v_{\perp}$, with mean zero. The statistical anisotropy of the dynamics is clear from the probability distributions of $v_{\|}$and $v_{\perp}$ in Figs. 1(c) and (d) respectively, which show a much greater dispersion along the rod than transverse to it 25]. Fig. 1(e) shows the distribution $P(\mathbf{v})$ of the two-dimensional velocity vector, peaked at a non-zero $v_{\|}$with significant weight in all directions including backwards. Our experiments can thus explore the applicability of the IFR in a much larger angular range than in the numerical study of [12]. Before presenting our experimental findings in detail, we build a minimal single-particle Langevin equation model for the dynamics of the two-dimensional position
$\mathbf{R}(t)=(X(t), Y(t))$ of the polar rod as a function of time $t$, ignoring inertia. This will allow an independent determination of parameters required later in the paper, and help relate our findings to the anisotropic [19] isometric fluctuation relation in a simplifying limit. As we work in a frame fixed in the particle, with $X$ and $Y$ defined with respect to the particle orientation, the dynamics of $\mathbf{n}_{\|}(t)$ does not enter our analysis. The governing Langevin equations read

$$
\boldsymbol{\Gamma} \cdot \dot{\mathbf{R}}=\mathbf{F}+\mathbf{N} \cdot \mathbf{f}(t)
$$

where $\mathbf{F}, \boldsymbol{\Gamma}$ are the systematic force and damping matrix. $\mathbf{F}$ gets contributions from the propulsive force driving the particle along $\mathbf{n}_{\|}(t)$ as well as from the spatial structure of the obstruction to motion posed by the bead medium. The random kicks that the rod receives from beads and plate are described by a noise $\mathbf{f}$. The physical noise covariance is $\mathbf{N} \cdot \mathbf{N}=2 \mathbf{D} \boldsymbol{\Gamma} \cdot \boldsymbol{\Gamma}$, which defines the diffusion tensor $\mathbf{D}$. We assume the unit-strength noise $\mathbf{f}$ to be Gaussian and white, a plausible assumption whose validity can be tested only by comparison to experiment. Our interest here, as in [12], is in large deviations of the macroscopic current $\int_{\mathbf{r}} \mathbf{J}(\mathbf{r}, t)$. For the present single-particle system the current density $\mathbf{J}(\mathbf{r}, t)=\delta(\mathbf{r}-\mathbf{R}(t)) \mathbf{v}(t)$ at point $\mathbf{r}$ at time $t$, where $\mathbf{v} \equiv \dot{\mathbf{R}}$, so that the macroscopic current is simply $\mathbf{v}(t)$.

We would like to extract the parameters in (10) from the experiment. We define $\mathbf{V}_{\tau}(t)=\tau^{-1} \int_{t}^{t+\tau} \mathbf{v}\left(t^{\prime}\right) d t^{\prime}$, the current coarse-grained on a timescale $\tau$. For the smallest accessible $\tau$, of order the inverse frame rate, it is reasonable to suppose that the structure of the bead medium does not affect the dynamics of the rod significantly. We can then assume $\mathbf{F}$ in (1) depends only on time. Define $\boldsymbol{\Pi} \equiv \mathbf{N}^{-1} \boldsymbol{\Gamma}, \mathbf{S} \equiv \mathbf{N}^{-1} \mathbf{F}$, and $\mathcal{S}_{\tau}(t)=\tau^{-1} \int_{t}^{t+\tau} \mathbf{S}\left(t^{\prime}\right) d t^{\prime}$, we can construct, from (1) and the statistics of the noise sources, the probability density

$$
P_{\tau}\left(\mathbf{V}_{\tau}=\mathbf{V}\right)=\operatorname{det} \boldsymbol{\Pi}\left(\frac{\tau}{2 \pi}\right)^{d / 2} \exp \left[-\frac{\tau}{2}\left(\boldsymbol{\Pi} \cdot \mathbf{V}-\mathcal{S}_{\tau}\right)^{2}\right]
$$

for $\mathbf{V}_{\tau}(t)$ to take a value $\mathbf{V}=\left(V_{\|}, V_{\perp}\right)$, via its momentgenerating function as shown in Appendix. If we approximate $\mathcal{S}_{\tau}(t)$ by $\boldsymbol{\Pi} \cdot \mathbf{v}_{0}$, where $\mathbf{v}_{0}$ is the steady-state average velocity, which would be exact for $\tau \rightarrow \infty$ and is reasonable for the present case where the inverse of frame rate, $\tau_{f}$, is four times the oscillation period, (2) becomes

$$
P_{\tau}\left(\mathbf{V}_{\tau}=\mathbf{V}\right)=(\operatorname{det} \mathbf{D})^{-1 / 2}\left(\frac{\tau}{2 \pi}\right)^{d / 2} e^{-\frac{\tau}{4}\left(\mathbf{V}-\mathbf{v}_{0}\right)^{T} \mathbf{D}^{-1}\left(\mathbf{V}-\mathbf{v}_{0}\right)}
$$

where the inverse diffusion tensor $(2 \mathbf{D})^{-1}=\boldsymbol{\Pi}^{T} \boldsymbol{\Pi}$ can be seen to provide a natural inner product. Below we use the form (3) to extract values for $v_{0}$ and the diffusivities from our data. Eq. (2), the result of ignoring the position dependence of the forcing in (1), trivially obeys an anisotropic IFR [19] because its large-deviation function, from (3), is quadratic: two coarse-grained currents 
$\mathbf{V}$ and $\mathbf{V}^{\prime}$ satisfying

$$
\mathbf{V}^{T} \mathbf{D}^{-1} \mathbf{V}=\mathbf{V}^{\prime T} \mathbf{D}^{-1} \mathbf{V}^{\prime}
$$

i.e., which lie on the ellipse $\mathbf{V}^{T} \mathbf{D}^{-1} \mathbf{V}=$ constant, obey

$$
\lim _{\tau \rightarrow \infty} \frac{1}{\tau} \ln \frac{P_{\tau}\left(\mathbf{V}_{\tau}=\mathbf{V}\right)}{P_{\tau}\left(\mathbf{V}_{\tau}=\mathbf{V}^{\prime}\right)}=\boldsymbol{\epsilon} \cdot\left(\mathbf{V}-\mathbf{V}^{\prime}\right) .
$$

with

$$
\boldsymbol{\epsilon}=\mathbf{v}_{0}^{T}(2 \mathbf{D})^{-1} .
$$

In the event that $D \Gamma$ turns out to be proportional to the unit tensor, with coefficient $T_{\text {eff }}$, local detailed balance holds. Then $\boldsymbol{\epsilon}=\boldsymbol{\Gamma} \mathbf{v}_{0} / 2 T_{\text {eff }}$ is the drag force scaled by effective temperature, and (5) becomes a true fluctuation relation for the power.

For comparison with our experiments, let us consider currents $\mathbf{V}, \mathbf{V}^{\prime}$ with equal magnitude $V$. The result of [12] can then be re-expressed, for the case of a diagonal $\mathbf{D}=\operatorname{diag}\left(D_{\|}, D_{\perp}\right)$, as

$$
\begin{gathered}
\lim _{\tau \rightarrow \infty} \frac{1}{\tau} \ln \frac{P_{\tau}\left(\mathbf{V}_{\tau}=\mathbf{V}\right)}{P_{\tau}\left(\mathbf{V}_{\tau}=\mathbf{V}^{\prime}\right)}=V\left[\frac{v_{0}^{\|}}{2 D_{\|}}\left(\cos \theta-\cos \theta^{\prime}\right)\right. \\
\left.+\frac{v_{0}^{\perp}}{2 D_{\perp}}\left(\sin \theta-\sin \theta^{\prime}\right)\right] \\
+\frac{V^{2}\left(D_{\|}-D_{\perp}\right)}{4 D_{\perp} D_{\|}}\left(\cos ^{2} \theta-\cos ^{2} \theta^{\prime}\right)
\end{gathered}
$$

Although the large-deviation function for our system will not have the quadratic form implied by (3), the foregoing calculation gives us a value [Eq. (6)] for $\epsilon$ in (5) in terms of independently measurable quantities. This allows a parameter-free comparison of our measurements to the Anisotropic Fluctuation Relation (AIFR) 19] in the form (5) or (7).

We return now to the experiments. The polar rod is propelled only along its nose, i.e., in the $x$ direction. Thus y-component of $\mathbf{F}$ in $(\mathbb{1})$ is zero, so that only the component $v_{0}^{\|}$of the mean velocity is nonzero. From the measured time series of positions we can extract the distribution of $\mathbf{V}_{\tau}$ for the shortest time accessible $\left(\tau_{f}\right)$ and infer $v_{0}^{\|}, D_{\|}$and $D_{\perp}$ by fitting the measured distribution to (3). We obtain $D_{\|}$and $D_{\perp}$ as $\left.\left\langle\left(V_{\|}(t)-v_{0}\right)^{2}\right\rangle_{t} \tau\right) / 2$ and $\left(\left\langle\left(V_{\perp}(t)\right)^{2}\right\rangle_{t} \tau\right) / 2$ respectively where $\tau=\tau_{f}=0.02 \mathrm{~s}$ and $v_{0}=0.34 \mathrm{~cm} \mathrm{~s}^{-1}$. We now plot constant velocity contours centered at $\left(V_{\|}=0, V_{\perp}\right.$ $=0$ ) and consider sets of $\mathbf{V}_{\tau}$ and $\mathbf{V}_{\tau}^{\prime}$ at angles $\theta$ and $\theta^{\prime}$ with respect to $\hat{\mathbf{n}_{\|}}$(Fig. 1(e)). We consider overlapping azimuthal bins of $\mathbf{V}_{\tau}$ in order to improve statistics, and obtain the probability density $P\left(\mathbf{V}_{\tau}\right)$. Defining $\Omega\left(\theta, \theta^{\prime}\right)=\frac{v_{0}^{\|}}{2 D_{\|}}+\frac{V\left(D_{\|}-D_{\perp}\right)}{4 D_{\perp} D_{\|}}\left(\cos \theta+\cos \theta^{\prime}\right)$, we plot $(\Omega \tau)^{-1} \ln \left[P_{\tau}(\mathbf{V}) / P_{\tau}\left(\mathbf{V}^{\prime}\right)\right]$ as a function of $\cos \theta-\cos \theta^{\prime}$,
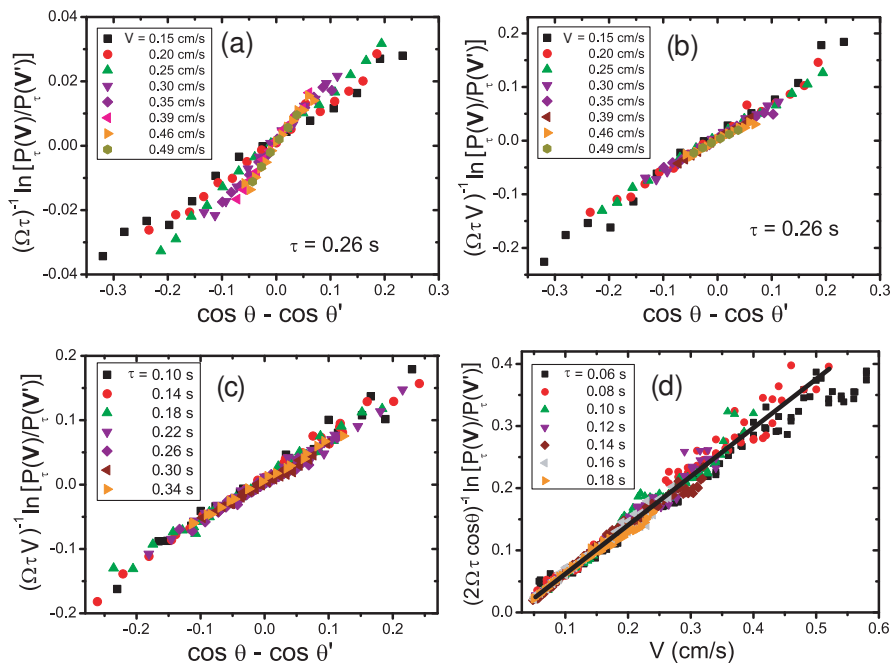

FIG. 2: (a) A typical plot of $(\Omega \tau)^{-1} \ln \left[P_{\tau}(\mathbf{V}) / P_{\tau}\left(\mathbf{V}^{\prime}\right)\right]$ vs. $\cos \theta-\cos \theta^{\prime}$ over various constant-velocity contours for $\tau=0.26 \mathrm{~s}$ showing a linear trend for all $V$. (b) Data scaling of $(\Omega \tau V)^{-1} \ln \left[P_{\tau}(\mathbf{V}) / P_{\tau}\left(\mathbf{V}^{\prime}\right)\right]$ vs. $\cos \theta-\cos \theta^{\prime}$.

(c) Scaling of $(\Omega \tau V)^{-1} \ln \left[P_{\tau}(\mathbf{V}) / P_{\tau}\left(\mathbf{V}^{\prime}\right)\right]$ with $\tau$ variation. Here each $\tau$ line contains all the $V$ values as in (b). (d) Plot of $(2 \Omega \tau \cos \theta)^{-1} \ln \left[P_{\tau}(\mathbf{V}) / P_{\tau}\left(\mathbf{V}^{\prime}\right)\right]$ vs $V$ for various $\tau$ for the special case when $\theta-\theta^{\prime}=180^{\circ}$. Here $\theta$ varies between $-30^{0}$ to $30^{\circ}$ in the step of $10^{0}$ for all $\tau$.

as shown in Fig. 2(a) for a typical $\tau=0.26 \mathrm{~s}$ at various values of $V$. The trend is linear for all $V$ in agreement with Eq. 7 (please recall that $v_{0}^{\perp}=0$ ). Furthermore, a plot of $(\Omega \tau V)^{-1} \ln \left[P_{\tau}(\mathbf{V}) / P_{\tau}\left(\mathbf{V}^{\prime}\right)\right]$ shows a clear scaling with $V$ for $\tau=0.26 \mathrm{~s}$ in Fig. 2(b). Similar data collapse is seen for all $\tau>0.10 \mathrm{~s}$. Now we plot $(\Omega \tau V)^{-1} \ln \left[P_{\tau}(\mathbf{V}) / P_{\tau}\left(\mathbf{V}^{\prime}\right)\right]$ for various $\tau$ in Fig. $2(\mathrm{c})$. Here each $\tau$ line contains various $V$ values as in Fig. 2(b). Overlapping lines for all $\tau$ confirm the $\tau$ scaling of the AIFR. From Eq. 7 the expected slope in Fig. 2(b) or (c) shold be unity. Our experiment, using the estimates for $v_{0}, D_{\|}$and $D_{\perp}$, finds a number close to 0.8. Perhaps this reflects limitations inherent in using estimates of mean speed and diffusivity from short-time data. A possible reason for this discrepancy is that the noise is not Gaussian [26]: Fig. 1(d) shows significant non-quadratic tails in the logarithm of the distribution of the small- $\tau$ averaged velocity, giving some credence to this suggestion.

As a check, we consider the special case of oppositely directed vectors, i.e. $\mathbf{V}^{\prime}=-\mathbf{V}$, (implying $\left.\theta-\theta^{\prime}=180^{\circ}\right)$. Fig. [2(d) shows the plot of $(2 \Omega \tau \cos \theta)^{-1} \ln \left[P_{\tau}(\mathbf{V}) / P_{\tau}\left(\mathbf{V}^{\prime}\right)\right]$ as a function of $V$ for various $\tau$. Here $\theta \in\left(-30^{0}, 30^{0}\right)$ separated by a step of $10^{\circ}$. A clean collapse of data is observed with the same slope as in Fig. 2(c). This illustrates compliance with the standard FR [1] as a special case of AIFR. 

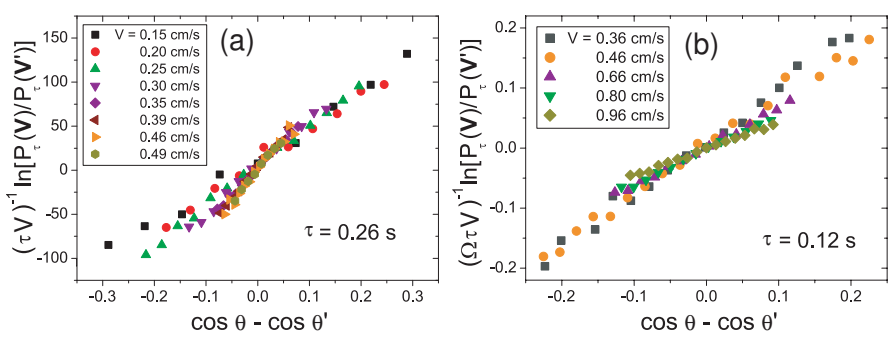

FIG. 3: (a) Ignoring the anistropy term in Eq. 7, i.e. assuming $D_{\|}=D_{\perp}$, no scaling is observed for $(\tau V)^{-1}$ $\ln \left[P_{\tau}(\mathbf{V}) / P_{\tau}\left(\mathbf{V}^{\prime}\right)\right]$ vs. $\cos \theta-\cos \theta^{\prime}$. (b) AIFR analysis for the particle moving on a bare substrate in the absence of bead medium. No collapse of data for $(\Omega \tau V)^{-1} \ln \left[P_{\tau}(\mathbf{V}) / P_{\tau}\left(\mathbf{V}^{\prime}\right)\right]$ vs. $\cos \theta-\cos \theta^{\prime}$ suggesting the presence of the noisy medium for AIFR to hold.

For completeness, we analyse the velocity fluctuations in the experiment in terms of the IFR for isotropic systems [12]. It can be obtained by setting $D_{\|}=D_{\perp}$ in Eq. 7 which gives $\Omega=v_{0} / 2 D_{\|}$independent of $\theta, \theta^{\prime}$. We plot $(\tau V)^{-1} \ln \left[P_{\tau}(\mathbf{V}) / P_{\tau}\left(\mathbf{V}^{\prime}\right)\right]$ against $\cos \theta-\cos \theta^{\prime}$ for $\tau=$ $0.26 \mathrm{~s}$. Fig. 3(a) shows that the isotropic IFR is not satisfied. We check that this trend is seen for all $\tau$. We find a trend of increasing slope with $V$ which cannot be explained without the inclusion of the second term in $\Omega$. This reiterates, experimentally, the importance of anisotropy as pointed out by [19]. We check in addition the role of the noise and hindrance to motion provided by the bead medium, by examining the velocity fluctuations of the particle for its motion on a bare substrate in the absence of any medium. Now the source of all the noise is multiple random collisions of the particle with the base and the lid. After an analysis along the same lines as above, we plot in Fig. [3(b) $(\Omega \tau V)^{-1} \ln \left[P_{\tau}(\mathbf{V}) / P_{\tau}\left(\mathbf{V}^{\prime}\right)\right]$ as function of $\cos \theta-\cos \theta^{\prime}$ for $\tau=0.12 s$. Interestingly, we find a significant failure of data-collapse in this case. Similar behaviour is observed for all $\tau$. The presence of the bead medium appears to be important for the AIFR to hold, a result consistent with our earlier findings [16].

It is curious that fluctuation relations, which are a derived consequence of microscopic time-reversibility, should arise in a system with a unidirectional flow of energy. It would appear that in the present experiment the only significant effect of this energy input is its transduction into directed motion by the polar rod. The central role of the bead medium is also a puzzle. We speculate the medium provides closely related processes governing the damping and diffusion matrices $\boldsymbol{\Gamma}$ and $\mathbf{D}$ in (1) of the polar rod, thus giving rise to an effective local detailed balance. In addition, perhaps the multiple collisions of the polar particle with the bead medium provides suppress correlated movements of the rod due to rolling or sliding, which may be present in motion on a bare substrate.
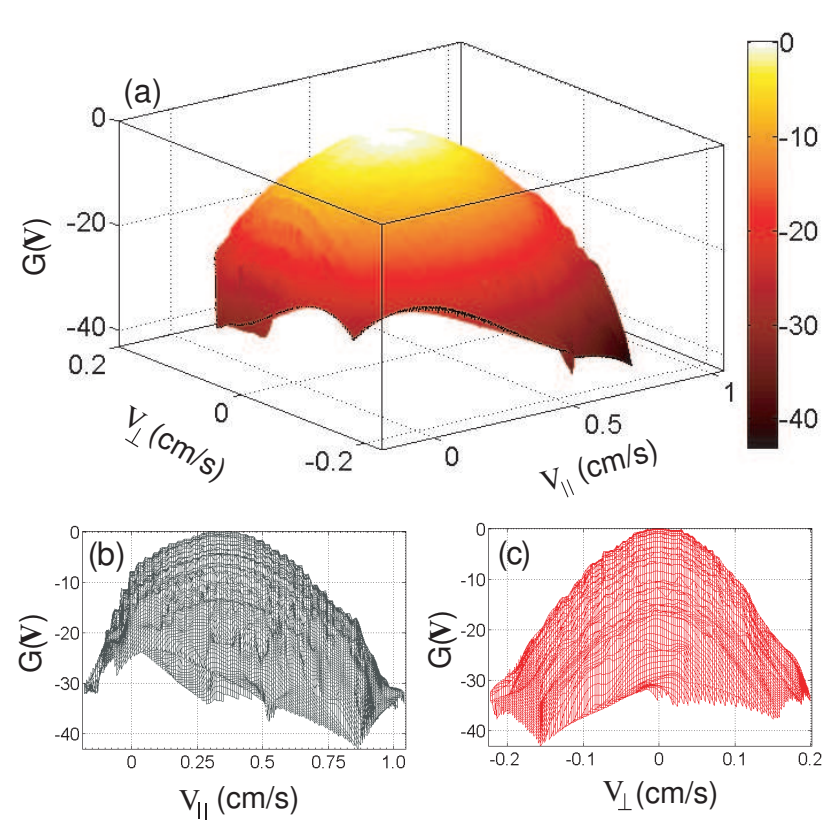

FIG. 4: (a) The Large Deviation Function for the velocity fluctuations for the case of the polar rod moving through the bead medium is non-paraboloid. (b) The side-view along the $V_{\perp}$-axis shows an abrupt fall which resembles the kink-like feature observed in [16]. (c) The side-view along the $V_{\|}$-axis.

Lastly, we extract the Large Deviation Function (LDF) for the velocity vector of a polar rod moving through the bead medium, $G(\mathbf{V})$ defined as $P_{\tau}(\mathbf{V})=A_{\tau} \exp (\tau G(\mathbf{V}))$ $(G(\mathbf{V})<0)$ in the limit $\tau \rightarrow \infty$ 27]. Here we take $A_{\tau}=\max \left[P_{\tau}(\mathbf{V})\right]=P_{\tau}\left(\langle\mathbf{V}\rangle=v_{0}\right)$ which is independent of $\mathbf{V}$ as required. We plot the asymptotic LDF as shown in Fig. U(a). Clearly the shape of LDF is far from paraboloid. There is a sharp drop in $G(\mathbf{V})$ below $V_{\|}=0$ plane which is clearer when viewed along the $V_{\perp}$-axis (Fig. 4(b)). This is related to the kink observed in earlier work [16]. Fig. 4(c) shows that the LDF is parabolic when viewed along the $V_{\perp}$ axis.

We point out here that earlier tests of the IFR, e.g., energy diffusion on a two-dimensional lattice, a harddisk fluid in a temperature gradient [12] were numerical. Moreover, these simulations saw no negative events and fluctuations were only along the imposed gradient, resulting in a test of the IFR in a very limited range of angles $\theta$. Our experimental study, with substantial noise, allows an exploration over a large $\theta$ range.

To summarise: we find detailed experimental support for the anisotropic variant [19] of the Isometric Fluctuation Relation [12], for a self-propelled granular particle moving through a monolayer of spherical beads. We suggest that the discrepancy between the observed and predicted prefactor arises from non-Gaussianity in the form of heavy tails in the microscopic noise, not accounted for 
in our Langevin equation model. More work is needed to address this. Our measurements are consistent with an earlier study of a Fluctuation Relation, and include an extension of our earlier observation of a kink in the LargeDeviation Function of the velocity vector of the particle. Our treatment through an anisotropic Langevin equation sheds some light on the circumstances in which such relations should arise, and allows a parameter-free test of the theory.

For support, NK thanks the University Grants Commission, India, HS thanks the CSIR, India, and AKS and SR acknowledge a J C Bose Fellowship of the DST, India.

\section{APPENDIX: Detailed calculation for Anisotropic isometric Fluctuation Relation}

The overdamped Langevin equations for a particle subjected to a force $\mathbf{F}(t)$ in $d$ dimensions can be written as

$$
\boldsymbol{\Gamma} \cdot \dot{\mathbf{R}}=\mathbf{F}+\mathbf{N} \cdot \mathbf{f}(t) .
$$

Multiplying above Eq. by $\mathbf{N}^{-1}$ gives

$$
\boldsymbol{\Pi} \cdot \dot{\mathbf{R}}=\mathbf{S}+\mathbf{f}(t),
$$

where $\boldsymbol{\Pi} \equiv \mathbf{N}^{-1} \boldsymbol{\Gamma}, \mathbf{S} \equiv \mathbf{N}^{-1} \mathbf{F}$. The current for the single particle at point $\mathbf{r}$ will be

$$
\mathbf{J}(\mathbf{r}, t)=\delta(\mathbf{r}-\mathbf{R}(t)) \mathbf{v}(t) .
$$

The macroscopic current averaged over time $\tau$ then reads

$$
\begin{aligned}
\mathbf{V}_{\tau}(t) & =\tau^{-1} \int_{t}^{t+\tau} \int_{\mathbf{r}} \mathbf{J}(\mathbf{r}, t) \\
& =\tau^{-1} \int_{t}^{t+\tau} \int_{\mathbf{r}} \delta\left(\mathbf{r}-\mathbf{R}\left(t^{\prime}\right)\right) \mathbf{v}\left(t^{\prime}\right) d t^{\prime} \\
& =\tau^{-1} \int_{t}^{t+\tau} \mathbf{v}\left(t^{\prime}\right) d t^{\prime}
\end{aligned}
$$

Average of the Eq. (9) over time duration $\tau$ gives

$$
\boldsymbol{\Pi} \cdot \mathbf{V}_{\tau}(t)=\mathcal{S}_{\tau}(t)+\mathcal{F}_{\tau}(t),
$$

where $\mathcal{S}_{\tau}(t)=\tau^{-1} \int_{t}^{t+\tau} \mathbf{S}\left(t^{\prime}\right) d t^{\prime}$ and $\mathcal{F}_{\tau}(t)=$ $\tau^{-1} \int_{t}^{t+\tau} \mathbf{f}\left(t^{\prime}\right) d t^{\prime}$. The probability density for $\mathcal{F}_{\tau}(t)$ can be written as

$$
\begin{gathered}
P\left(\mathcal{F}_{\tau}(t)=\mathbf{A}\right)=\left\langle\delta\left(\frac{1}{\tau} \int_{t}^{t+\tau} \mathbf{f}\left(t^{\prime}\right) d t^{\prime}-\mathbf{A}\right)\right\rangle_{\mathbf{f}} \\
=\frac{1}{(2 \pi)^{d}}\left\langle\int_{\mathbf{k}} \exp -i\left(\frac{1}{\tau} \int_{t}^{t+\tau} \mathbf{k} \cdot \mathbf{f}\left(t^{\prime}\right) d t^{\prime}-\mathbf{k} \cdot \mathbf{A}\right)\right\rangle_{\mathbf{f}} \\
=\frac{1}{(2 \pi)^{d}} \int_{\mathbf{k}}\left\langle\exp -i \frac{1}{\tau} \int_{t}^{t+\tau} \mathbf{k} \cdot \mathbf{f}\left(t^{\prime}\right) d t^{\prime}\right\rangle_{\mathbf{f}} \exp i \mathbf{k} \cdot \mathbf{A}(13)
\end{gathered}
$$

But from a well known identity [28]

$$
\left\langle\exp \left(\int_{0}^{\tau} H(t) g(t) d t\right)\right\rangle_{g}=\exp \left(\frac{1}{2} \int_{0}^{\tau} H(t)^{2} d t\right)
$$

for any arbitrary function $H(t)$ and any white Gaussian noise $g(t)$ with zero mean and deviation one,

$$
\begin{aligned}
P\left(\mathcal{F}_{\tau}(t)=\mathbf{A}\right) & =\frac{1}{(2 \pi)^{d}} \int_{\mathbf{k}} \exp \left(-\frac{1}{2 \tau^{2}} \int_{0}^{\tau} k^{2} d t+i \mathbf{k} \cdot \mathbf{A}\right) \\
& =\frac{1}{(2 \pi)^{d}} \int_{\mathbf{k}} \exp \left(-\frac{k^{2}}{2 \tau}+i \mathbf{k} \cdot \mathbf{A}\right) .
\end{aligned}
$$

RHS of above equation is just Inverse Fourier Transform of a Gaussian function exp $-k^{2} / 2 \tau$, therefore the probability density for $\mathcal{F}_{\tau}(t)$ becomes

$$
P\left(\mathcal{F}_{\tau}(t)=\mathbf{A}\right)=\left(\frac{\tau}{2 \pi}\right)^{d / 2} \exp \left(-\frac{\tau A^{2}}{2}\right) .
$$

From Jacobian transformation the probability density for $\mathbf{V}_{\tau}(t)$ is

$$
P\left(\mathbf{V}_{\tau}(t)\right)=\operatorname{det}\left(\frac{\partial \mathcal{F}_{\tau}}{\partial \mathbf{V}_{\tau}}\right) P\left(\mathcal{F}_{\tau}(t)\right) .
$$

But from Eq. (12)

$$
\frac{\partial \mathcal{F}_{\tau}}{\partial \mathbf{V}_{\tau}}=\boldsymbol{\Pi},
$$

hence

$$
\begin{aligned}
P\left(\mathbf{V}_{\tau}(t)=\right. & \mathbf{V})=\operatorname{det} \boldsymbol{\Pi}\left(\frac{\tau}{2 \pi}\right)^{d / 2} \times \\
& \exp \left(-\frac{\tau}{2}\left(\boldsymbol{\Pi} \cdot \mathbf{V}-\mathcal{S}_{\tau}(t)\right)^{2}\right) .
\end{aligned}
$$

Since $\boldsymbol{\Pi}^{T} \boldsymbol{\Pi}=(2 \mathbf{D})^{-1}$,

$$
\begin{aligned}
\operatorname{det} \boldsymbol{\Pi} & =\left(\operatorname{det}\left(2 \mathbf{D}^{-1}\right)\right)^{1 / 2} \\
& =2^{-d / 2}(\operatorname{det} \mathbf{D})^{-1 / 2}
\end{aligned}
$$

and

$$
\begin{aligned}
& \left(\boldsymbol{\Pi} \cdot \mathbf{V}-\mathcal{S}_{\tau}(t)\right)^{2} \\
& =\left(\boldsymbol{\Pi} \cdot \mathbf{V}-\mathcal{S}_{\tau}(t)\right)^{T}\left(\boldsymbol{\Pi} \cdot \mathbf{V}-\mathcal{S}_{\tau}(t)\right) \\
& =\left(\mathbf{V}-\boldsymbol{\Pi}^{-1} \cdot \mathcal{S}_{\tau}(t)\right)^{T} \boldsymbol{\Pi}^{T} \boldsymbol{\Pi}\left(\mathbf{V}-\boldsymbol{\Pi}^{-1} \mathcal{S}_{\tau}(t)\right) \\
& =\frac{1}{2}\left(\mathbf{V}-\boldsymbol{\Pi}^{-1} \cdot \mathcal{S}_{\tau}(t)\right)^{T} \mathbf{D}^{-1}\left(\mathbf{V}-\boldsymbol{\Pi}^{-1} \mathcal{S}_{\tau}(t)\right)(.20)
\end{aligned}
$$

Eq. (19) then becomes

$$
\begin{array}{r}
P_{\tau}\left(\mathbf{V}_{\tau}=\mathbf{V}\right)=(\operatorname{det} \mathbf{D})^{-1 / 2}\left(\frac{\tau}{4 \pi}\right)^{d / 2} \times \\
e^{-\frac{\tau}{4}\left(\mathbf{V}-\boldsymbol{\Pi}^{-1} \cdot \mathcal{S}_{\tau}(t)\right)^{T} \mathbf{D}^{-1}\left(\mathbf{V}-\boldsymbol{\Pi}^{-1} \cdot \mathcal{S}_{\tau}(t)\right)} .
\end{array}
$$

If the average velocity of the particle in steady state is $\mathbf{v}_{0}$, the $\mathcal{S}_{\tau}(t)$ can be approximated by $\boldsymbol{\Pi} \cdot \mathbf{v}_{0}$, thus probability density for $\mathbf{V}_{\tau}(t)$ becomes

$$
\begin{aligned}
P_{\tau}\left(\mathbf{V}_{\tau}=\mathbf{V}\right)= & (\operatorname{det} \mathbf{D})^{-1 / 2}\left(\frac{\tau}{4 \pi}\right)^{d / 2} \times \\
& e^{-\frac{\tau}{4}\left(\mathbf{V}-\mathbf{v}_{0}\right)^{T} \mathbf{D}^{-1}\left(\mathbf{V}-\mathbf{v}_{0}\right)} .
\end{aligned}
$$




\section{In 2 dimensions}

In two dimensions, for diagonal $\mathbf{D}=\operatorname{diag}\left(D_{\|}, D_{\perp}\right), \mathrm{Eq}$. (22) becomes

$$
\begin{aligned}
P_{\tau}\left(\mathbf{V}_{\tau}=\mathbf{V}\right) & =\frac{\tau}{4 \pi \sqrt{D_{\|} D_{\perp}}} \times \\
& \exp \left[-\frac{\tau\left(V_{\|}-v_{0}^{\|}\right)^{2}}{4 D_{\|}}-\frac{\tau\left(V_{\perp}-v_{0}^{\perp}\right)^{2}}{4 D_{\perp}}\right]
\end{aligned}
$$

Two coarse-grained currents $V$ and $V^{\prime}$ satisfying the condition

$$
\frac{V_{\|}^{2}}{D_{\|}}+\frac{V_{\perp}^{2}}{D_{\perp}}=\frac{V_{\|}^{\prime 2}}{D_{\|}}+\frac{V_{\perp}^{\prime 2}}{D_{\perp}}
$$

i.e., which lie on the ellipse $V_{\|}^{2} / D_{\|}+V_{\perp}^{2} / D_{\perp}=$ constant, obey

$$
\lim _{\tau \rightarrow \infty} \frac{1}{\tau} \ln \frac{P_{\tau}\left(\mathbf{V}_{\tau}=\mathbf{V}\right)}{P_{\tau}\left(\mathbf{V}_{\tau}=\mathbf{V}^{\prime}\right)}=\boldsymbol{\epsilon} \cdot\left(\mathbf{V}-\mathbf{V}^{\prime}\right)
$$

For currents $\mathbf{V}, \mathbf{V}^{\prime}$ with equal magnitude $V$ the result of [12] can be re-expressed as

$$
\begin{aligned}
& \lim _{\tau \rightarrow \infty} \frac{1}{\tau} \ln \frac{P_{\tau}\left(\mathbf{V}_{\tau}=\mathbf{V}\right)}{P_{\tau}\left(\mathbf{V}_{\tau}=\mathbf{V}^{\prime}\right)} \\
= & V\left[\frac{v_{0}^{\|}}{2 D_{\|}}\left(\cos \theta-\cos \theta^{\prime}\right)+\frac{v_{0}^{\perp}}{2 D_{\perp}}\left(\sin \theta-\sin \theta^{\prime}\right)\right] \\
+ & \frac{V^{2}\left(D_{\|}-D_{\perp}\right)}{4 D_{\perp} D_{\|}}\left(\cos ^{2} \theta-\cos ^{2} \theta^{\prime}\right)
\end{aligned}
$$

The true large-deviation function will presumably not have the quadratic form implied by (23). One should note that this calculation gives us a value

$$
\boldsymbol{\epsilon}=\left(v_{0}^{\|} / 2 D_{\|}, v_{0}^{\perp} / 2 D_{\perp}\right)
$$

for $\epsilon$ in (25) in terms of independently measurable quantities.

* On leave from the Department of Physics, Indian Institute of Science, Bangalore

[1] D.J. Evans and D.J. Searles, Adv. Phys. 51, 1529-1585 (2002); G Gallavotti, Eur. Phys. J. B 61, 1-24 (2008); U. Seifert, Eur. Phys. J. B 64, 423-431 (2008).
[2] N. Garnier and S. Ciliberto, Phys. Rev. E 71, 060101 (2005).

[3] W. I. Goldburg et al., Phys. Rev. Lett. 87, 245502 (2001).

[4] F. Douarche, S. Ciliberto, and A. Petrosyan, J. Stat. Mech. (2005) P09011.

[5] F. Douarche, S. Joubaud, N. B. Garnier, A. Petrosyan, and S. Ciliberto, Phys. Rev. Lett. 97, 140603 (2006).

[6] S Ciliberto, N Garnier, S Hernandez, C Lacpatia, J.-F Pinton, G Ruiz Chavarria, Physica A 340, 240 (2004).

[7] K. Feitosa and N. Menon, Phys. Rev. Lett. 92, 164301 (2004).

[8] A. Puglisi, P. Visco, A. Barrat, E. Trizac, and F. van Wijland, Phys. Rev. Lett. 95, 110202 (2005).

[9] S. Majumdar and A. K. Sood, Phys. Rev. Lett. 101, 078301 (2008).

[10] S. Majumdar and A. K. Sood, Phys. Rev. E. 85, 041404 (2012).

[11] S. K. Nandi et al., J. Stat. Mech, 2013, P02027 (2013).

[12] P. I. Hurtado et al., Proc. Nat. Acad. Sci. (USA) 78, 641-692 (2011).

[13] D. Yamada, T. Hondou, and M. Sano, Phys. Rev. E 67, 040301(R) (2003).

[14] V. Narayan, S. Ramaswamy, N. Menon, Science 317, 105-8 (2007).

[15] A. Kudrolli, G. Lumay, D. Volfson, and L.S. Tsimring, Phys. Rev. Lett. 100, 058001 (2008).

[16] N. Kumar, S. Ramaswamy, and A. K. Sood, Phys. Rev. Lett. 106, 118001 (2011).

[17] N. Kumar, H. Soni, S. Ramaswamy and A.K. Sood, Nat. Commun. 54688 doi: 10.1038/ncomms5688 (2014).

[18] Although our system is not obviously reversible at the level of the dynamics of rod and bead coordinates, with a one-way mean flux of energy from the shaker into unresolved internal modes and ultimately into radiant heat, the results of [16] prompt this further exploration.

[19] R. Villavicencio et al., Europhys. Lett. 105, 30009 (2014)

[20] W.S. Rasband, ImageJ, U. S. National Institutes of Health, Bethesda, Maryland, USA, http://rsb.info.nih.gov/ij/, 1997-2009.

[21] J. Mehl, T. Speck and U. Seifert, Phys. Rev. E 78, 011123 (2008).

[22] K. Mallick , J. Stat. Mech. P01024 (2011).

[23] D.Lacoste, A. W.C. Lau and K. Mallick, Phys. Rev. E 78, 011915 (2008).

[24] A.A. Budini, Phys. Rev. E 84, 061118 (2011).

[25] In view of our remark above on the time-binning implicit in the definition of $\mathbf{V}(t)$, this is not necessarily evidence for two temperatures.

[26] Perhaps this is because rolling motion, with some degree of persistence and hence fat tails, contributes to the lateral movement of the rod.

[27] H. Touchette, Phys. Rep. 478, 1-69 (2009).

[28] V. Balakrishnan, Pramana Journal of Physics 40, 259 (1993). 